\title{
3D Analysis of the Sound Reduction Provided by Protective Surfaces Around a Noise Source
}

\author{
Luís Godinho \\ CICC, Department of Civil Engineering, University of Coimbra, Coimbra, Portugal
}

\author{
Edmundo G. A. Costa and José A. F. Santiago \\ COPPE/Federal University of Rio de Janeiro, Rio de Janeiro, Brazil
}

\author{
Andreia Pereira and Paulo Amado-Mendes \\ CICC, Department of Civil Engineering, University of Coimbra, Coimbra, Portugal
}

\begin{abstract}
(Received 12 July 2012; provisionally accepted 23 June 2013; accepted 20 August 2013)
In the present paper, the authors present a numerical analysis of the sound reduction provided by simple acoustic protective measures to attenuate the noise emitted by equipment placed near a façade. The noise source is assumed to be surrounded by an enclosed space, defined as a parallelepiped chamber with a rectangular opening. To perform the numerical analysis, a 3D Boundary Element formulation is implemented. This formulation makes use of the domain decomposition, together with Green's functions, specifically defined to reduce the size of the involved system matrices and to allow consideration for surface absorption. Indeed, these Green's functions, defined using the image-source technique, allow modelling of the building's façade and the ground as infinite surfaces with a given absorption coefficient. The numerical model is verified against an analytical solution known for the case of a point load acting within a parallelepiped space; additionally, it is validated by comparing its results with those obtained experimentally for a simple case. The implemented model is then used to perform a number of numerical simulations, illustrating the effect of different configurations of the protective surfaces in the sound reduction.
\end{abstract}

\section{INTRODUCTION}

Many numerical methods have been used to model sound propagation in both 2D and 3D environments. During the last four decades, the Boundary Element Method (BEM) has established itself as one of the preferred methods to be used in acoustics and vibro-acoustics engineering analysis. In fact, the BEM has a number of advantages over other numerical methods that contribute to its success, as noted by Brebbia. ${ }^{1}$ First, it only requires the discretization of the problem boundaries, and thus only involves a more compact description of the environment. Second, it has a very good accuracy, since it is based on the use of Greens functions, which are, themselves, a solution of the governing equation. Finally, it is very well suited to the analysis of infinite or semi-infinite domains, as the farfield radiation conditions are automatically satisfied. Some of these advantages are even more pronounced when a 3D analysis in an infinite/semi-infinite domain is considered, for which alternative methods frequently require millions of degrees of freedom, together with a truncation of the propagation domain and the use of approximate absorbing boundary conditions.

Through the years, many researchers have used the method in acoustic analysis of different systems. Many resources for the BEM can be found, such as the excellent books by $\mathrm{Wu}^{2}$ and Von Estorff, ${ }^{3}$ which describe the fundamentals of the boundary element based acoustic analysis. Interesting developments can be found in many scientific papers, such as the early works of Lacerda et al., ${ }^{4}$ in which a dual BEM formulation is used to analyse the 2D sound propagation around acoustic barriers, over an infinite plane, considering both the ground and the barrier to be absorptive. Later, the $3 \mathrm{D}$ propagation of sound around an absorptive barrier has been studied by Lacerda et al., ${ }^{5}$ introducing a dual boundary element formulation that allowed the barrier to be modelled as a simple surface. Several studies were also published concerning the convergence and the discretization requirements of the BEM, such as the works of Tadeu et al., ${ }^{6}$ Tadeu and António, ${ }^{7}$ or Marburg. ${ }^{8}$ Many recent works have also focused on the development of the BEM and its variants for complex acoustic problems, including more intricate acoustic models. For example, these have been applied to silencer or baffle analysis ${ }^{9-11}$ and have been used to propose highly efficient numerical approaches to tackle largescale problems. ${ }^{12,13}$

Perhaps the strongest drawbacks of the BEM are its complex mathematical formulation and the fact that it requires the prior knowledge of fundamental solutions, which are available only for some specific types of differential equations with specific boundary conditions. However, for acoustic spaces, with typically homogeneous propagation media, those solutions are well known, and the method has been applied with success. ${ }^{2,3}$ Another point that should not be neglected is that the accuracy of a BEM model depends on the special treatment of analytical and numerical integration of the singular and hypersingular integrals. This treatment is not trivial in the general case, and specific numerical integration strategies need to be devised in some cases to allow for accurately performing those integrations (as it is the case of the strategy devised in Telles ${ }^{14}$ and Telles and Oliveira ${ }^{15}$ ). Recently, a new strategy based on a closed form integration of singular and hypersingular integrals 